\title{
Postoperative Intraspinal Subdural Collections after Pediatric Posterior Fossa Tumor Resection: Incidence, Imaging, and Clinical Features
}

\author{
J.H. Harreld, N. Mohammed, G. Goldsberry, X. Li, Y. Li, F. Boop, and Z. Patay
}

o- $\equiv$

\begin{abstract}
BACKGROUND AND PURPOSE: Postoperative intraspinal subdural collections in children after posterior fossa tumor resection may temporarily hinder metastasis detection by MR imaging or CSF analysis, potentially impacting therapy. We investigated the incidence, imaging and clinical features, predisposing factors, and time course of these collections after posterior fossa tumor resection.
\end{abstract}

MATERIALS AND METHODS: Retrospective review of postoperative spine MRI in 243 children (5.5 \pm 4.6 years of age) from our clinical data base postresection of posterior fossa tumors from October 1994 to August 2010 yielded 37 (6.0 \pm 4.8 years of age) subjects positive for postoperative intraspinal subdural collections. Their extent and signal properties were recorded for postoperative (37/37), preoperative (15/37), and follow-up spine (35/37) MRI. Risk factors were compared with age-matched internal controls ( $n=37,5.9 \pm 4.5$ years of age). Associations of histology, hydrocephalus and cerebellar tonsillar herniation, and postoperative intracranial subdural collections with postoperative intraspinal subdural collections were assessed by the Fisher exact test or $\chi^{2}$ test. The association between preoperative tumor volume and postoperative intraspinal subdural collections was assessed by the Wilcoxon rank sum test.

RESULTS: The overall incidence of postoperative intraspinal subdural collections was 37/243 (15.2\%), greatest $\leq 7$ days postoperatively (36\%); $97 \%$ were seen $0-41$ days postoperatively (12.9 \pm 11.0 days). They were T2 hyperintense and isointense to CSF on TIWI, homogeneously enhanced, and resolved on follow-up MR imaging (35/35). None were symptomatic. They were associated with intracranial subdural collections $(P=.0011)$ and preoperative tonsillar herniation $(P=.0228)$.

CONCLUSIONS: Postoperative intraspinal subdural collections are infrequent and clinically silent, resolve spontaneously, and have a distinctive appearance. Preoperative tonsillar herniation appears to be a predisposing factor. In this series, repeat MR imaging by 4 weeks documented improvement or resolution of these collections in $88 \%$.

ABBREVIATIONS: PISC $=$ postoperative intraspinal subdural collections; PISC $+=$ positive for postoperative intraspinal subdural collections; POD $=$ postoperative day

$\mathrm{T}$ he importance of MR imaging for the diagnosis of leptomeningeal metastasis in children with posterior fossa tumors, critical for risk stratification and treatment, ${ }^{1}$ is well-recognized. MR imaging detects leptomeningeal metastasis in up to $50 \%$ of those with false-negative CSF examination findings, which may occur in up to $45 \%$ of initial lumbar punctures, ${ }^{2}$ and findings on MR imaging correlate better with survival than CSF results. ${ }^{2-4} \mathrm{Al}-$

Received September 26, 2014; accepted after revision November 1.

From the Departments of Radiological Sciences (J.H.H., Z.P.), Biostatistics (X.L., Y.L.), and Surgery (F.B.), St. Jude Children's Research Hospital, Memphis, Tennessee; Department of Imaging Diagnostic (N.M.), Institute of Paediatric, Kuala Lumpur Hospital, Kuala Lumpur, Malaysia; and Department of Radiology (G.G.), McFarland Clinic, Ames, lowa.

This work was supported in part by grant No. CA21765 from the National Cancer Institute and by the American Lebanese Syrian Associated Charities.

Paper previously presented in part at: Annual Meeting of the American Society of Neuroradiology and the Foundation of the ASNR Symposium, April 21-26, 2012; New York, New York. though preoperative brain and spine MR imaging are optimal, neurosurgical urgency may dictate deferral until the postoperative period, or repeat spine imaging may be necessary to exclude leptomeningeal metastasis postoperatively. A few case reports and small series have described spinal subdural collections and enhancement on postoperative myelography and MR imaging. ${ }^{5-8}$ These findings were further investigated in a larger series by Warmuth-Metz et al, ${ }^{9}$ who described such findings in children after posterior fossa (7/45) but not supratentorial (0/8) tumor resection. However, due to the limited availability of follow-up imaging, the natural history could not be established.

\footnotetext{
Please address correspondence to Zoltan Patay, MD, PhD, St. Jude Children's Research Hospital, Department of Radiological Sciences, 262 Danny Thomas Place, MS-220, Memphis, TN 38105; e-mail: zoltan.patay@stjude.org

- Indicates open access to non-subscribers at www.ajnr.org

三 Indicates article with supplemental on-line table.

http://dx.doi.org/10.3174/ajnr.A4221
} 
At St. Jude Children's Research Hospital, enrollment of patients with posterior fossa tumors on long-term therapeutic protocols confers the advantage of follow-up imaging availability. Because most arrive postoperatively and undergo subsequent metastasis-screening MR imaging according to our imaging protocols before enrollment, we see a number of postoperative intraspinal subdural collections (PISC). While these collections do not indicate metastasis, ${ }^{6,7,9}$ they may be misinterpreted by radiologists unfamiliar with their appearance, prompt neurosurgical consult, or hinder metastasis detection in the critical postoperative staging period. We therefore investigated the incidence, imaging and clinical features and course, and associated potentially predisposing factors in patients with PISC after suboccipital tumor resection in a large pediatric cohort.

\section{MATERIALS AND METHODS Study Population}

A retrospective Health Insurance Portability and Accountability Act-compliant, institutional review board-approved clinical data base search conducted with a waiver of consent yielded 304 subjects who underwent resection of posterior fossa tumors between October 1994 and August 2010. Of these, 243 subjects (age range, 1 month to 20 years; mean age, $5.5 \pm 4.6$ years; $40.3 \%$ female; 139 medulloblastomas, 69 ependymomas, 15 atypical teratoid rhabdoid tumors, 5 astrocytomas, 3 glioblastomas, 3 gliomas, 1 medullomyoblastoma, 1 primitive neuroectodermal tumor, 7 rhabdoid tumors) had available postoperative spine MR imaging of sufficient diagnostic quality for detection of PISC. MR imaging review yielded 37 subjects with evidence of PISC (age range, 6 months to 16 years; mean, $6.0 \pm 4.8$ years; $37.8 \%$ female; 24 medulloblastomas, 7 ependymomas, 2 astrocytomas, 4 atypical teratoid rhabdoid tumors). Medical records were reviewed for CSF findings and for evidence of neurologic signs and symptoms related to irritation or compression of intraspinal neural structures at the time of MR imaging. To exclude the influence of age, we then established an age-matched internal control group negative for PISC ( $n=37$; age range, 5 months to 16.6 years; mean, $5.9 \pm 4.5$ years; $35 \%$ female; 27 medulloblastomas, 8 ependymomas, 2 atypical teratoid rhabdoid tumors) for comparative risk factor analysis.

\section{Spine MR Imaging Analysis}

Spine MR imaging techniques varied due to the 16-year study time span and multi-institutional imaging protocols. All spine MRIs were performed for metastasis screening and included postcontrast T1WI. All MRIs were reviewed by a board-certified neuroradiologist with a Certificate of Added Qualification (J.H.H., with 4.5 years' experience interpreting pediatric spine MR imaging). A second board-certified neuroradiologist (Z.P., with 18 years' experience interpreting pediatric spine MR imaging) reviewed all MRI in the final cohort (those positive for postoperative subdural collections [PISC+ ] and controls negative for postoperative subdural collection), equivocal cases, and those ultimately excluded for technical reasons. Differences were resolved in consensus. Both reviewers were blinded to pathology and radiology reports. MRIs of subjects deemed PISC + on the first postoperative MR imaging or on any MR imaging $\leq 30$ days after surgery were followed until they were negative for PISC. Time from surgery to spine MR imaging was recorded for all examinations. The presence, location (cervical, thoracic, lumbosacral), extent (expressed in number of spinal segments), maximum thickness, and signal properties of PISC were assessed on sagittal postcontrast T1WI (37/37 subjects positive for PISC), precontrast T1WI (13/37) and T2WI (20/37) on postoperative MRIs, and subsequent follow-up spine MRIs in 35/37 subjects positive for PISC; follow-up spine MRIs were unavailable in 2/37 subjects positive for PISC. Signal on T1WI and T2WI was defined as isointense, hyperintense, or hypointense to CSF in the perimedullary subarachnoid space. Preoperative spine MRIs available in 15/37 subjects positive for PISC were assessed for the presence of subdural collections. Original reports from referring institutions were subsequently reviewed.

\section{Brain MR Imaging Analysis}

Preoperative brain MRIs of all subjects were evaluated for the location (fourth ventricle or not) and size of the posterior fossa tumor and for hydrocephalus by both subjective assessment and Evans index $>90$ th percentile. ${ }^{10}$ Tonsillar herniation was defined as $>5-\mathrm{mm}$ protrusion of the cerebellar tonsils below the foramen magnum. Incomplete preoperative brain imaging for 1 subject positive for PISC precluded evaluation for tonsillar herniation or Evans index. Tumor volumes were calculated, per ellipsoid volume, as

$$
V=\frac{4}{3} \pi \times \frac{L}{2} \times \frac{W}{2} \times \frac{H}{2} .
$$

Postoperative brain imaging, CT in 1 subject and MRI in 36/37 subjects, were evaluated for intracranial subdural collections (supratentorial or infratentorial) as visualized on T1WI without (36/ 36 ) and with contrast (31/36), T2WI (35/36), noncontrast FLAIR (26/36), and contrast-enhanced FLAIR (18/36) images. Intracranial subdural collections were subjectively graded as nonexistent (0), mild (1), moderate (2), or large (3).

\section{Statistical Analysis}

Statistical analyses were performed by using SAS 9.3 software (SAS Institute, Cary, North Carolina). Associations of PISC with tumor histology and grade of intracranial subdural collection were assessed by the Fisher exact test. Association of PISC with tumor volume was evaluated by the Wilcoxon rank sum test. The associations of PISC with tumor location, subjective assessment of hydrocephalus, Evans index $>90$ th percentile, and the presence of tonsillar herniation and intracranial subdural collection were evaluated by the $\chi^{2}$ test. Age and time from surgery to MR imaging were compared between all subjects positive and negative for postoperative subdural collections by the Wilcoxon rank sum test. $P<.05$ was considered statistically significant.

\section{RESULTS}

The overall incidence of PISC was 37/243 (15.2\%). In the overall cohort, subjects positive for PISC were imaged earlier $(18 \pm 26$ days postoperatively) than those without PISC ( $30 \pm 32$ days postoperatively) $(P=.0005)$, and the incidence rate of PISC fell from $36 \%$ for subjects imaged up to 7 days after surgery to $8 \%$ in 
Percentage risk of PISC in entire cohort $(n=243)$ by number of days from PFS to MRI

\begin{tabular}{lccc}
\hline $\begin{array}{c}\text { PFS to } \\
\text { MRI (days) }\end{array}$ & PISC+ & $\begin{array}{c}\text { Total No. } \\
\text { Subjects }\end{array}$ & $\%$ PISC+ \\
\hline $0-7$ & 15 & 42 & $36 \%$ \\
$8-14$ & 6 & 44 & $14 \%$ \\
$15-21$ & 6 & 48 & $13 \%$ \\
$22-28$ & 5 & 43 & $11 \%$ \\
$>28$ & 5 & 66 & $8 \%$ \\
\hline
\end{tabular}

Note:-PFS indicates posterior fossa surgery.

those imaged after postoperative day (POD) 28 (Table). There was no association of PISC with age $(P=.5033)$.

Compared with controls, subjects positive for PISC did not differ significantly in age $(P=.9957)$ or time from surgery to MR imaging $(P=.0723)$. There was no association between PISC and tumor histology $(P=.4573)$. Chart review yielded no neurologic signs or symptoms related to irritation or compression of intraspinal neural structures contemporaneous with the diagnosis of PISC.

\section{Time to Appearance}

The time from posterior fossa surgery to the first postoperative MR imaging revealing PISC ranged from 0 to 41 days (mean, $15 \pm$ 17 days) in 36/37 (97\%) subjects and was 137 days in a single subject (On-line Table). The time from posterior fossa surgery to the first postoperative MR imaging (mean, $17.6 \pm 10.8$ days) was 1-49 days in controls. Two subjects with PISC 27 days postoperatively had earlier negative MRI findings on PODs 11 and 20, respectively. No subdural collections were visible on preoperative MRI available in 15/37 subjects positive for PISC.

\section{Time to Resolution}

Further follow-up MRIs were available in 35/37 subjects positive for PISC. In 34 subjects, MR imaging demonstrated resolution of PISC between 8 and 472 days (108.2 \pm 123.4 days) postoperatively, which was 3-441 days (95 \pm 119.5 days) after the first PISC+ MR imaging. In another subject who had residual unresectable ependymoma at the foramen magnum and was diagnosed with PISC on POD 137, PISC persisted on MR imaging 325 days postoperatively. Resolution was documented 5.9 years after surgery, at which time the primary tumor had decreased in size so that the foramen magnum was patent. Otherwise, PISC had resolved in $100 \%$ of subjects re-imaged $\geq 57$ days after the initial PISC+ MR imaging and had resolved in 12/17 (70.5\%) subjects re-imaged between 3 and 28 days after the initial PISC+ MR imaging (On-line Table).

Three subjects with PISC diagnosed 1, 12, and 27 days after surgery had persistent but decreased collections on follow-up MR imaging 17, 6, and 28 days later (PODs 18, 18, and 55), respectively (On-line Table); all had resolved on repeat MR images obtained 35, 84, and 30 days later (PODs 53, 102, and 85), respectively. In 1 subject with PISC 3 days after surgery, PISC persisted at 17 days, with no further follow-up.

\section{Location and Size}

Two subjects were excluded from location and size analysis due to imaging limited to the cervical and thoracolumbar spine, respectively, precluding full analysis despite demonstrating PISC. Sub- dural collections were most frequently thoracic (33/35), followed by lumbosacral (29/35) and cervical (27/35). PISC spanned an average of $18.8 \pm 8.7$ vertebral bodies at initial diagnosis, and except for a single subject with only ventral collections, all were both dorsal and ventral. PISC measured up to $0.46 \pm 0.1 \mathrm{~mm}$ in thickness ventrally and $0.43 \pm 0.21 \mathrm{~mm}$ dorsally, with maximal thickness most frequently at lumbar levels ventrally (19/35) and dorsally (18/35).

\section{Imaging Characteristics}

PISC were isointense to CSF on noncontrast T1WI (13/13) (Fig $1)$, hyperintense $(19 / 20)$ more frequently than isointense to CSF (1/20) on T2WI, and most frequently hyperintense (enhancing) on postcontrast T1WI (36/37), on which they were most readily visualized (Figs 1 and 2). PISC were isointense to CSF on postcontrast T1WI in 1 subject. They were lobulated on sagittal postcontrast T1WI and T2WI and on axial imaging when available (Fig 2). No hematocrit levels were observed. There was no evidence of associated cord deformity or signal abnormality suggestive of compression in any subject, though prominent lumbar PISC often caused central crowding of the cauda equina without compression (Fig 2).

\section{Associated Imaging Findings}

PISC were not associated with preoperative hydrocephalus by either subjective assessment $(P=.0749)$ or by Evans index $>90 \%$ $(P=.7222)$. There was no significant relationship between either tumor volume $(P=.8797)$ or fourth ventricular location $(P=$ .3594) and the development of PISC.

PISC were significantly associated with preoperative cerebellar tonsillar herniation $(P=.0228)$ and with both the presence $(P=$ $.0011)$ and magnitude $(P=.0071)$ of postoperative intracranial subdural collections; $91.89 \%$ of patients with PISC had intracranial subdural collections, compared with $59.46 \%$ of controls. Intracranial collections were most frequently mild in both subjects positive for PISC (67.57\%) and control subjects (43.24\%). Intracranial collections were more frequently associated with tonsillar herniation in subjects positive for PISC than in controls $(P=$ .0116).

\section{Correlation with CSF}

CSF was obtained an average of 5 days ( \pm 13 days) after the first postoperative MR imaging in 29/37 subjects, 146 days later in 1 subject, 392 days later in another, and unavailable in 6/37. Seven subjects had spinal metastases visible on PISC + MR imaging; CSF was positive in $2 / 7$, both medulloblastomas. In 1 case, metastases were questionable until resolution of PISC. In another, metastases were previously diagnosed on preoperative MR imaging; CSF was negative for metastases.

In another medulloblastoma case, PISC + MR imaging and same-day CSF were negative for metastasis, despite CSF positive for metastasis 2 days later. Fluid color was not recorded.

Six subjects underwent lumbar puncture immediately following PISC + MR imaging negative for metastasis. Collected fluid was yellow (xanthochromic) in 2/6 and negative for malignant cells in $6 / 6$.

AJNR Am J Neuroradiol 36:993-99 May 2015 www.ajnr.org 

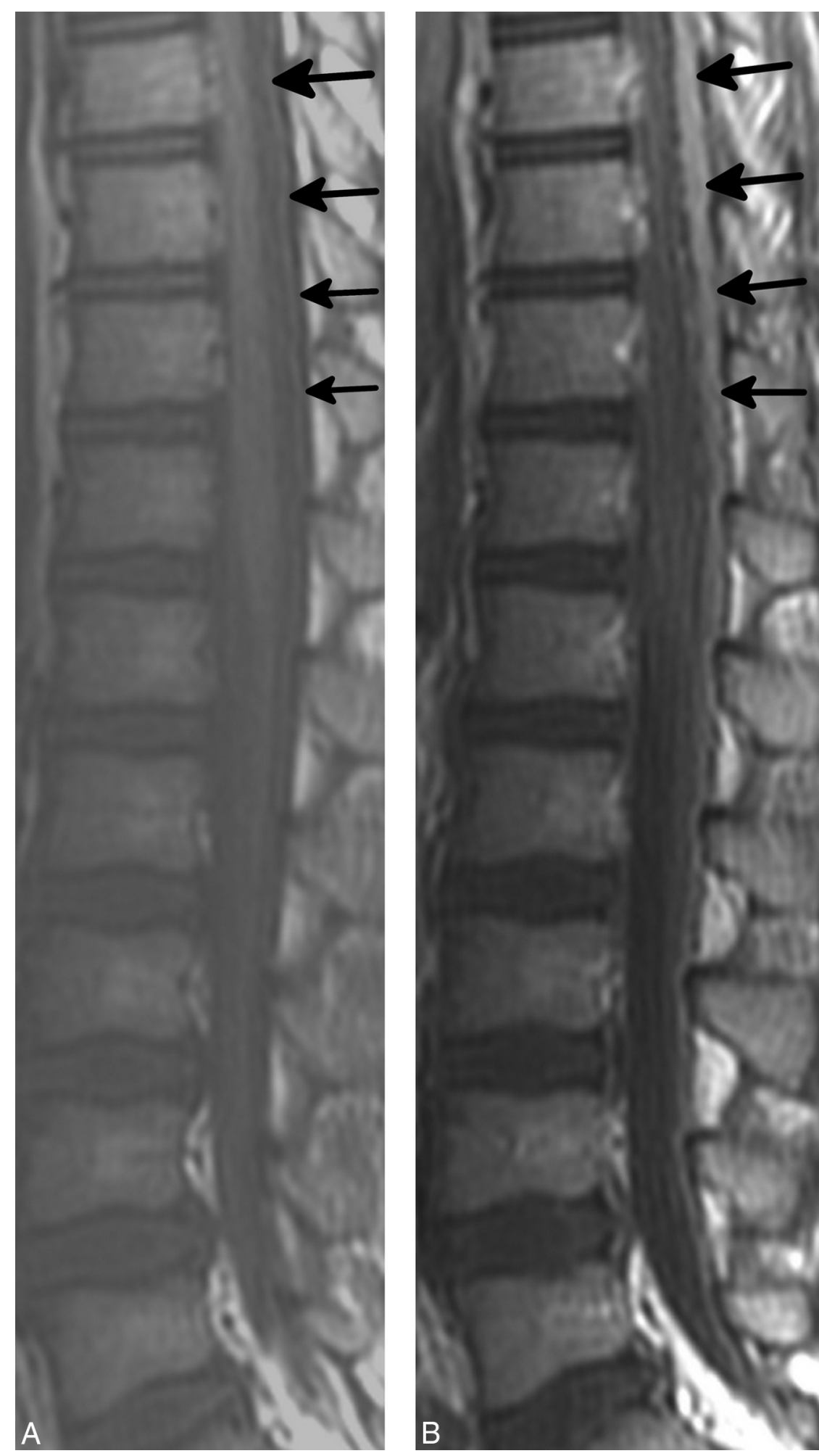

FIG 1. PISC (arrows) appear isointense to CSF on TIWI (A) and enhance on postcontrast TIWI (B).

\section{Diagnosis}

PISC were correctly identified on 23/23 MRIs interpreted by radiologists employed by St. Jude Children's Research Hospital, a pediatric cancer specialty hospital. Two PISC + examinations performed at St. Jude were interpreted by locum tenens radiologists; PISC were incorrectly interpreted as potential metastases in both instances. Of 12 examinations performed and interpreted at outside referring institutions, PISC were missed in 3, misinterpreted as contrast leakage in 1 , misinterpreted as metastasis or potential metastasis in 4 , and correctly diagnosed in $4 / 12$. Thus, of 14 examinations interpreted by nonspecialized radiologists, only $4(28.6 \%)$ were correctly interpreted as PISC, and 6/14 (42.9\%) were misinterpreted as metastasis or potential metastasis.

\section{DISCUSSION}

Enhancing spinal subdural collections have been described in $15.5 \%-23 \%$ of children after posterior fossa surgery, ${ }^{7,9}$ but descriptions of associated factors and natural history have been limited due to sample size and follow-up limitations. In this study, we found PISC in 37 (15.2\%) of 243 children imaged after posterior fossa surgery, similar to the $15.5 \%$ frequency described by Warmuth-Metz et al. ${ }^{9}$ PISC were associated with both intracranial subdural collections and tonsillar herniation. Although relief of increased intracranial pressure as evidenced by preoperative obstructive hydrocephalus has been suggested as a mechanism, ${ }^{9}$ we found no association of PISC with hydrocephalus either by subjective assessment or by the Evans index. $^{10}$

PISC appeared as late as $>20$ days postoperatively, resolved as early as 8 days after surgery, and had resolved in most $(70.5 \%)$ subjects imaged up to 28 days after initial PISC+ MR imaging. The incidence of PISC is greatest, $36 \%$ in this series, within 1 week after surgery and drops precipitously thereafter. In a single subject with unresectable ependymoma partially obstructing the foramen magnum and leptomeningeal metastasis, PISC persisted for at least 325 days; resolution was documented 5 years after surgery, at which time the primary tumor had decreased in size so that the foramen magnum was again patent. Although the collections were frequently large, significantly effacing CSF, there was no imaging or clinical evidence of compression of spinal neural elements. However, PISC did partially obscure metastases in at least 1 case, resulting in a questionable diagnosis, and may have been sampled rather than CSF to give a false-negative CSF result on lumbar puncture in at least 1 patient who had positive lumbar puncture findings 2 days later. Xanthochromic fluid sampled in 2 additional subjects may 

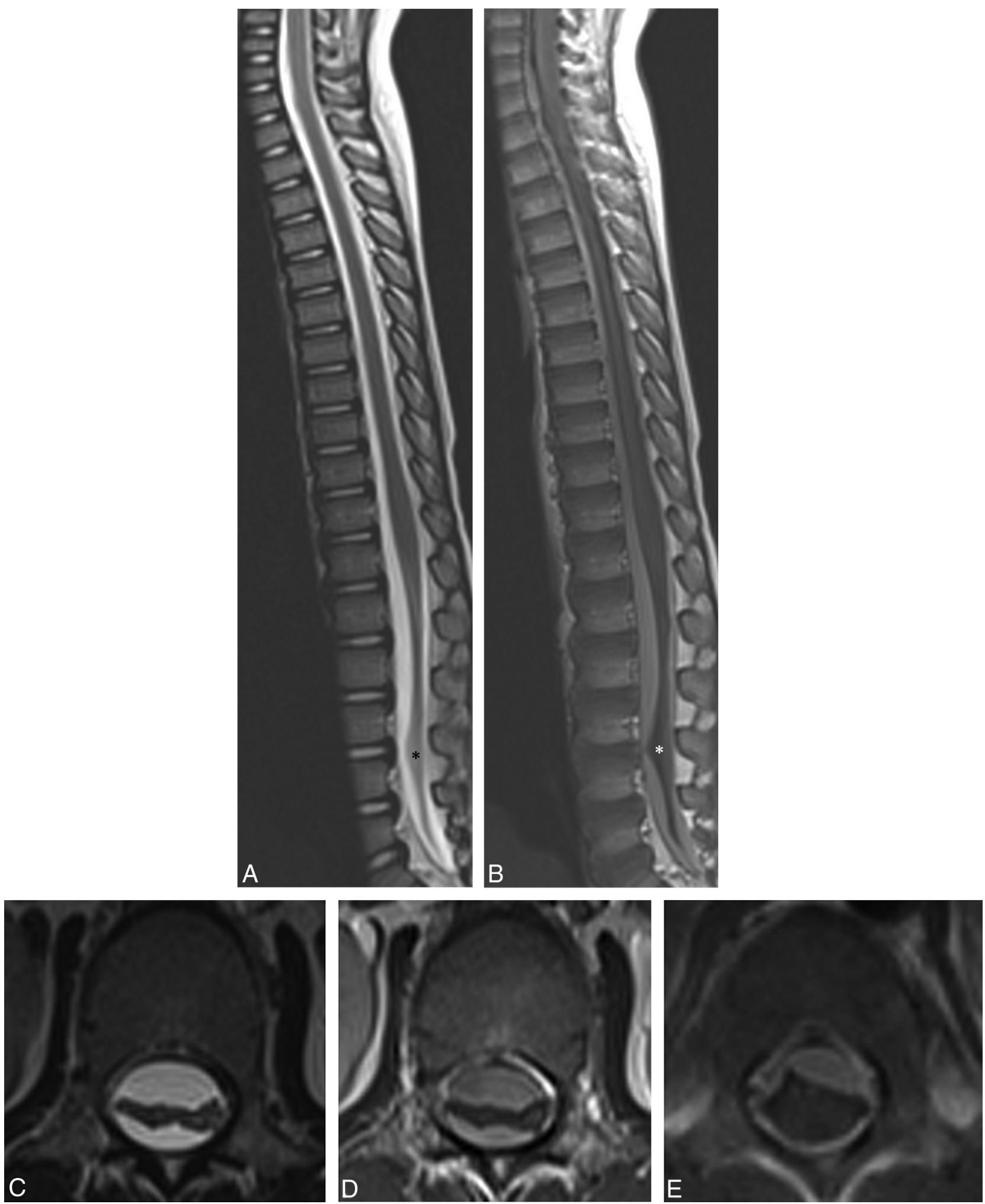

FIG 2. PISC filling the spinal canal appear mildly hyperintense to CSF (asterisk) on sagittal T2WI $(A)$ and enhance on postcontrast TIWI (B), POD 10. PISC centrally displace the cauda equina on axial T2WI $(C)$ and postcontrast TIWI (D) in another subject, POD 12. Festooned, enhancing PISC persist on postcontrast TIWI (E) in another subject on POD 41.

also have been subdural rather than CSF. ${ }^{11}$ Although even lumbar punctures performed under ideal conditions may be falsely negative in up to $45 \%,{ }^{2}$ these observations suggest that PISC may render the diagnosis of metastasis more challenging on both MR imaging and CSF examinations; this problem potentially results in incorrect risk stratification and treatment of the primary pathology. Of equal importance is the potential for misdiagnosis of PISC as metastasis by radiologists unfamiliar with this phenomenon, as occurred in $42.9 \%$ of spine MRI interpreted by nonspecialized radiologists in this series, potentially resulting in unnec- 
essarily aggressive therapy and attendant increases in associated risks.

Thirty-six of 37 (97.3\%) PISC were homogeneously hyperintense on postcontrast T1WI in this series, a characteristic finding. Although most subjects had only high-resolution postcontrast MR imaging as per our institutional leptomeningeal metastasis screening protocol, isointensity to CSF on noncontrast T1WI confirmed enhancement in 13/37, consistent with findings in prior reports. ${ }^{6,7,9}$ Despite this enhancement, the subdural location and lobulated appearance readily distinguish PISC from leptomeningeal metastases, which are subarachnoid in location and typically nodular, though they may diffusely "sugarcoat" the spinal cord. ${ }^{1}$

The appearance of PISC is similar to that of spinal subdural collections in patients with spontaneous intracranial hypotension, including a "festooned" appearance on axial imaging due to attachments spanning the "subdural" zone (Fig 2), supporting decreased CSF pressure as a common mechanism. ${ }^{12-14}$ The Monro-Kellie hypothesis, which states that the sum of volumes of intracranial blood, CSF, and tissue must remain constant in an intact cranium, is generally invoked to explain the intracranial subdural collections as compensating for low CSF volumes in spontaneous intracranial hypotension or for sudden loss of intracranial volume postoperatively. ${ }^{12,14-18}$ This hypothesis has been extended to account for intraspinal subdural collections in spontaneous intracranial hypotension and after posterior fossa surgery. ${ }^{9,19}$

It has been suggested that microtears in the arachnoid layer due to low CSF pressure and retraction of the arachnoid from the dura permit leakage of CSF into the subdural space; ${ }^{18-20}$ however, such freely communicating CSF collections, termed "hygromas," do not demonstrate the enhancement observed in PISC. 6,9,11,21 PISC are more consistent with subdural effusions, which are encapsulated collections of protein-rich, xanthochromatic fluid not in communication with the subarachnoid space; enhancement differentiates them from hygromas. ${ }^{21}$ In actuality, there is no true "subdural space"; subdural effusions form within a relatively weak vascularized fibroblast cell layer, known as the "dural border" cell layer, at the interface of the dura and arachnoid layers, ${ }^{13,14,21}$ due to negative hydrostatic pressure inducing vasodilation and increased permeability in the dural border cell layer, with leakage of plasma and contrast resulting in enhancing subdural effusions. ${ }^{6,9,11}$

Of all subjects with intracranial subdural collections, cerebellar tonsillar herniation was more frequent in those with PISC, an association not previously described. We therefore hypothesize that sequestration of the intraspinal and intracranial spaces, rather than increased intracranial pressure alone, predisposes patients with tonsillar herniation to PISC via relatively increased intraspinal pressure via a "ball valve" mechanism, followed by an abrupt postoperative pressure decrease. The requirement for compartmental sequestration could explain the absence of PISC in children with supratentorial tumors, which are less commonly associated with tonsillar herniation, though intracranial subdural collections may be present within the same supratentorial compartment postshunting or after decompressive craniotomy. ${ }^{17,18}$

Limitations of this study include irregular timing of follow-up spine MRI, occurring up to $>1$ year after diagnosis of PISC, limiting precise evaluation of the natural history. However, nearly all patients in this series, the largest to date, had follow-up imaging documenting resolution of PISC, and it would be difficult to justify administering contrast and anesthesia to asymptomatic patients for MR imaging for this purpose prospectively. Formal neurologic examinations were not performed because the patients were asymptomatic. CSF pressure measurements are not routinely obtained at lumbar puncture for metastasis detection at St. Jude Children's Research Hospital, precluding correlation with PISC. Although xanthochromic and false-negative fluid was obtained at lumbar puncture in 3 subjects, subdural fluid was not intentionally sampled; this sampling could be informative in future studies.

\section{CONCLUSIONS}

Enhancing postoperative intraspinal subdural effusions occurred in $15.2 \%$ of children after posterior fossa tumor resection in this series, resolved spontaneously without neurologic sequelae, and had a characteristic appearance. Abrupt postoperative relief of excessive intraspinal pressure caused by spinal sequestration by tonsillar herniation is the proposed etiology. Preoperative spine MR imaging is ideal, but not always possible. Because time is of the essence for establishing a diagnosis of metastasis, delaying postoperative imaging, as previously suggested, ${ }^{6}$ is not advised because PISC occur infrequently and metastases may be visible despite PISC. However, we do recommend that if subdural collections are detected, MR imaging with contrast be repeated as late as clinically tolerated to re-evaluate leptomeningeal metastasis and potentially confounding lumbar PISC before lumbar puncture. In this series, repeat MR imaging documented improvement or resolution of PISC in $88 \%$ by 4 weeks.

Disclosures: Julie H. Harreld—UNRELATED: Grants/Grants Pending: National Cancer Institute, * American Lebanese Syrian Associated Charities, ${ }^{*}$ Comments: St. Jude Children's Research Hospital is a Comprehensive Cancer Center receiving grant support from the National Cancer Institute, grant No. CA21765. American Lebanese Syrian Associated Charities is the fundraising arm of St. Jude Children's Research Hospital and supports all clinical and research activities; Travel/Accommodations/Meeting Expenses Unrelated to Activities Listed: Society of Pediatric Radiology; World Federation of Neuroradiological Societies, Comments: Society of Pediatric Radiology supported travel expenses and honorarium for an invited talk in 2013. World Federation of Neuroradiological Societies supported travel expenses for an invited talk in 2014. Frederick Boop-RELATED: Consulting Fee or Honorarium: Vancouver Children's Hospital*; UNRELATED: Board Membership: Association of Neurological Surgeons*; Employment: Semmes-Murphey Clinic; OTHER RELATIONSHIPS: Board Member; Chairman, Department of Neurosurgery, University of Tennessee Health Science Center. Zoltan Patay-UNRELATED: Consultancy: Guerbet, Comments: one-time advisory board participation in 2013; Grants/Grants Pending: American Lebanese Syrian Associated Charities, ${ }^{*}$ National Cancer Institute CA21765*; Travel/ Accommodations/Meeting Expenses Unrelated to Activities Listed: Symposium Neuroradiologicum (Turkey), Third International Congress of NeuroOncology (Algeria), Seventh Arab Radiology Congress (Qatar), Hungarian Neuroradiology Society (Hungary), The Hospital for Sick Children (Canada), Indian Society of Neuroradiology (India), Sao Paulo Radiology Meeting (Brasil), Kuwait International Neuroimaging Conference (Kuwait), Erasmus Course of Magnetic Resonance Imaging (Romania, Belgium), European Course of Pediatric Neuroradiology (Italy), Comments: invited lectures given at mentioned meetings and courses. I received reimbursement of travel and lodging expenses. *Money paid to the institution.

\section{REFERENCES}

1. Engelhard HH, Corsten LA. Leptomeningeal metastasis of primary central nervous system (CNS) neoplasms. Cancer Treat Res 2005;125:71-85 
2. Maroldi R, Ambrosi C, Farina D. Metastatic disease of the brain: extra-axial metastases (skull, dura, leptomeningeal) and tumour spread. Eur Radiol 2005;15:617-26

3. Terterov S, Krieger MD, Bowen I, et al. Evaluation of intracranial cerebrospinal fluid cytology in staging pediatric medulloblastomas, supratentorial primitive neuroectodermal tumors, and ependymomas. J Neurosurg Pediatr 2010;6:131-36

4. Pang J, Banerjee A, Tihan T. The value of tandem CSF/MRI evaluation for predicting disseminated disease in childhood central nervous system neoplasms. J Neurooncol 2008;87:97-102

5. Porto L, Kieslich M, Turowski B, et al. Spinal subdural haematoma mimicking tethered cord after posterior fossa open surgery. Neuroradiology 2002;44:415-17

6. Shaw DW, Weinberger E, Brewer DK, et al. Spinal subdural enhancement after suboccipital craniectomy. AJNR Am J Neuroradiol 1996; 17:1373-77

7. Kaufman BA, Moran CJ, Park TS. Spinal magnetic resonance imaging immediately after craniotomy for detection of metastatic disease. Pediatr Neurosurg 1995;23:171-81

8. Wiener MD, Boyko OB, Friedman HS, et al. False-positive spinal MR findings for subarachnoid spread of primary CNS tumor in postoperative pediatric patients. AJNR Am J Neuroradiol 1990;11:1100-03

9. Warmuth-Metz M, Kuhl J, Krauss J, et al. Subdural enhancement on postoperative spinal MRI after resection of posterior cranial fossa tumours. Neuroradiology 2004;46:219-23

10. Wilk R, Kluczewska E, Syc B, et al. Normative values for selected linear indices of the intracranial fluid spaces based on CT images of the head in children. Pol J Radiol 2011;76:16-25

11. Teksam M, Casey SO, McKinney A, et al. Gadolinium enhancement of spinal subdural collection on magnetic resonance imaging after lumbar puncture. Neuroradiology 2003;45:553-56
12. Chiapparini L, Ciceri E, Nappini S, et al. Headache and intracranial hypotension: neuroradiological findings. Neurol Sci 2004;25(suppl 3):S138-41

13. Haines DE, Harkey HL, al-Mefty O. The "subdural" space: a new look at an outdated concept. Neurosurgery 1993;32:111-20

14. Mokri B, Parisi JE, Scheithauer BW, et al. Meningeal biopsy in intracranial hypotension: meningeal enhancement on MRI. Neurology 1995;45:1801-07

15. Mokri B. The Monro-Kellie hypothesis: applications in CSF volume depletion. Neurology 2001;56:1746-48

16. Tosaka M, Sato N, Fujimaki H, et al. Diffuse pachymeningeal hyperintensity and subdural effusion/hematoma detected by fluid-attenuated inversion recovery MR imaging in patients with spontaneous intracranial hypotension. AJNR Am J Neuroradiol 2008;29:1164-70

17. Aarabi B, Chesler D, Maulucci C, et al. Dynamics of subdural hygroma following decompressive craniectomy: a comparative study. Neurosurg Focus 2009;26:E8

18. Sonmez OF, Temel Y, Visser-Vandewalle V, et al. A new evaluation method for the intracranial volume changes and subdural effusion of patients following endoscopic third ventriculostomy. Clin Neurol Neurosurg 2013;115:160-64

19. Schievink WI. Spontaneous spinal cerebrospinal fluid leaks and intracranial hypotension. JAMA 2006;295:2286-96

20. Watanabe A, Horikoshi T, Uchida M, et al. Diagnostic value of spinal MR imaging in spontaneous intracranial hypotension syndrome. AJNR Am J Neuroradiol 2009;30:147-51

21. Mori K, Maeda M. Delayed magnetic resonance imaging with GdDDTPA differentiates subdural hygroma and subdural effusion. Surg Neurol 2000;53:303-10; discussion 310-11 